\title{
Foreground effect on the $J$-factor estimation of classical dwarf spheroidal galaxies
}

\author{
Koji Ichikawa, ${ }^{(a)}$ Miho N. Ishigaki, ${ }^{(a)}$ Shigeki Matsumoto, ${ }^{(a)}$ Masahiro Ibe, ${ }^{(a, b)}$ \\ Hajime Sugai, ${ }^{(a)}$ Kohei Hayashi, ${ }^{(a, c)}$ and Shun-ichi Horigome ${ }^{(a)}$ \\ (a) Kavli IPMU (WPI), UTIAS, The University of Tokyo, Kashiwa, 277-8583, Japan \\ (b) ICRR, The University of Tokyo, Kashiwa, 277-8582, Japan \\ (c) Kavli Institute for Astronomy and Astrohysics, Peking University, Beijing 100871, China
}

Accepted XXX. Received YYY; in original form ZZZ

\begin{abstract}
The gamma-ray observation of the dwarf spheroidal galaxies (dSphs) is a promising approach to search for the dark matter annihilation (or decay) signal. The dSphs are the nearby satellite galaxies with a clean environment and dense dark matter halo so that they give stringent constraints on the $O(1) \mathrm{TeV}$ dark matter. However, recent studies have revealed that current estimation of astrophysical factors relevant for the dark matter searches are not conservative, where the various non-negligible systematic uncertainties are not taken into account. Among them, the effect of foreground stars on the astrophysical factors has not been paid much attention, which becomes more important for deeper and wider stellar surveys in the future. In this article, we assess the effects of the foreground contamination by generating the mock samples of stars and using a model of future spectrographs. We investigate various data cuts to optimize the quality of the data and find that the cuts on the velocity and surface gravity can efficiently eliminate the contamination. We also propose a new likelihood function which includes the foreground distribution function. We apply this likelihood function to the fit of the three types of the mock data (Ursa Minor, Draco with large dark matter halo, and Draco with small halo) and three cases of the observation. The likelihood successfully reproduces the input $J$-factor value while the fit without considering the foreground distribution gives large deviation from the input value by a factor of three.
\end{abstract}

Key words: galaxies: dwarf spheroidals- galaxies:kinematics and dynamics $-\gamma$-rays: galaxies-instrumentation: spectrographs-dark matter -astroparticle physics

\section{INTRODUCTION}

The existence of dark matter is solidly confirmed by various observations such as the dynamics of galaxy clusters (Zwicky 1933), rotation curves of the galaxies (Rubin, Thonnard \& Ford 1978; Rubin, Ford \& . Thonnard 1980) and gravitational lensing (McLaughlin 1999; Lokas \& Mamon 2003; Clowe et al. 2006; Bradac et al. 2006) as well as the global fit of the Cosmic Microwave Background (CMB), Large Scale Structure (LSS), and Supernovae (SNs) observations (Ade et al. 2016). Its identity, however, has remained unknown for almost eighty years since its first postulation. The identification of dark matter is certainly one of the most important tasks in cosmology, astrophysics, and particle physics. Despite our limited knowledge of its nature, it is strongly expected that dark matter is not a part of the standard model of the elementary particle physics. Among various candidates for dark matter, weakly interacting massive particle (WIMP) is considered to be one of the most attractive candidates. The thermally produced WIMP dark matter can explain the observed dark matter density naturally by its annihilation into lighter particles in the standard model. In fact, the preferred mass range of the WIMP is $\lesssim O(1) \mathrm{TeV}$ since otherwise its annihilation rate is suppressed and the mass density becomes inconsistent with the observation. With this successful feature, the WIMP dark matter has gathered particular attention in conjunction with the physics beyond the standard model such as supersymmetry (see e.g. Jungman, Kamionkowski \& Griest (1996) also Murayama (2007); Feng (2010)).

Indirect detection experiment, which aims to observe the signals induced by the dark matter annihilation, is one of the most important searches for the WIMP dark matter because it has the strong sensitivity to the most interesting mass scale, i.e., $O(1) \mathrm{TeV}$. In particular, the gamma-ray search is a promising approach because it is free from the 
propagation uncertainty and the detection capability is sufficiently large. Many astronomical objects potentially are to be targets of the gamma-ray dark matter detection. Among them, dwarf spheroidal satellite galaxies of the Milky Way (dSphs) are ideal targets because they are nearby galaxies $(\sim 10-100 \mathrm{kpc}$ from the solar system) at high latitude with dense dark matter halo. ${ }^{1}$ In fact, current observations have given stringent constraints on the dark matter annihilation cross sections by searching for the gamma-ray signal of dark matter (Ackermann et al. 2015) from dSphs. As pointed out in Bhattacherjee et al. (2014), however, the constraints crucially depend on the astrophysical uncertainties of the dark halo shape of the dSphs especially the ones of the so-called ultra-faint dSphs whose numbers of the observed stars are less than $O(100)$. Recent detailed analyses have also revealed hidden but non-negligible uncertainties of the dark matter profile: prior bias (Martinez et al. 2009), anisotropy of the velocity dispersion (Ullio \& Valli 2016), size of the halo truncation (Geringer-Sameth et al. 2015), non-sphericity (Bonnivard et al. 2015b; Hayashi et al. 2016), and foreground contamination (Bonnivard et al. 2016). ${ }^{2}$

These systematic uncertainties are partly caused by the lack of sufficiently large stellar kinematic data available from current spectroscopic instruments. For the eight luminous dSphs (so-called classical dSphs), discovered before the Sloan Digital Sky Survey project (York et al. 2000), Walker et al. (2009c) used line-of-sight velocity measurements of up to a few thousand stars in each galaxy to constrain their dark matter content, based on the observations with the Michigan/MIKE Fibre System at the Magellan 6.5 $\mathrm{m}$ Clay telescope and the Hectochelle spectrograph at the $6.5 \mathrm{~m}$ Multiple Mirror Telescope. The number of stars is limited by their limiting magnitudes ( $i$-band magnitudes of up to 19.5) and/or the small field-of-view compared to the tidal radii of these galaxies. For the ultra-faint dSphs, the number of stars is intrinsically small and thus even with a multi-object spectrograph mounted on a $8-10 \mathrm{~m}$ class telescope (e.g. Deep Imaging Multi-Object Spectrograph at Keck telescope), the velocity measurements for only a few tens to a few hundred stars are available (Simon \& Geha 2007; Simon et al. 2011; Kirby et al. 2013; Koposov et al. 2011; Aden et al. 2009; Walker et al. 2009b; Simon et al.

1 The observation of the galactic centre of the Milky Way is also sensitive to the dark matter signal. However, the signal flux highly depends on the shape of the dark matter profile and understanding of the astrophysical background is difficult. The most conservative estimation is weaker than that of dSphs.

2 The existence of the unresolved binary stars can also have impact on the estimation of the velocity dispersion. For classical dSphs, although the typical fractions lie around 20 $60 \%$, the effect of these binary stars is negligible because their intrinsic velocity dispersions are much larger than those of the binary stars (Olszewski et al. 1996; Walker et al. 2006; Mateo, Olszewski \& Walker 2008; Koch et al. 2007; Minor 2013). For some ultra-faint dSphs, the velocity variability due to the unresolved binary stars is measured and it is confirmed that the binary effect does not significantly inflate the velocity dispersion (Simon \& Geha 2007; Simon et al. 2011; McConnachie \& Cote 2010; Koposov et al. 2011; Kirby et al. 2013; Simon et al. 2015b). However, one should make clear this effect when one use dataset of a new ultra-faint dSph, which will be resolved by the multiepoch velocity data. 2015b; Simon et al. 2015a; Martin et al. 2016; Kirby et al. 2015). However, since most of the member stars exist below their sensitivity $(i \sim 21)$, the number of the measured velocities is about $O(10-100)$.

Most of the uncertainties mentioned above would be reduced with future wide-field multi-object spectrographs mounted on large telescopes that are capable of efficiently covering the outer regions of $\mathrm{dSphs}$ and reaching fainter magnitudes (e.g. $i>21.0$ ). However, the spectroscopic data always includes the foreground stars which belong to the Milky Way galaxy. Such contamination will remain problematic or even become worse in the future observations. Current approach to eliminate the foreground stars is to calculate their membership probability, which is obtained through the expectation maximization process using radial velocity, projected position, and metallicities (Walker et al. 2009a). However, once the stars with high membership probability are chosen, they are usually considered as the true member stars and the effect of the residual foreground stars is ignored in the halo profile estimation. As Bonnivard et al. (2016) have pointed out, this contamination can derive a non-negligible overestimation of $J$-factor and therefore more detailed analysis of the foreground contamination is required for the future detailed kinematical survey.

In this paper, we investigate the foreground effect in the future observation. The data cuts which minimize the fraction of the foreground stars are investigated by using mock data under a realistic setup of the future spectrographic observation, which provides the prospect of the number and quality of the future stellar data at the same time. We also construct a new method for the $J$-factor estimation convolving the foreground contamination. This method is compared with the usual halo estimation which gives largely biased $J$ factor by the foreground contamination. The organization of this paper is as follows. In section 2, we introduce a formula of the signal flux and clarify the uncertainties which are not taken into accounted in the current analysis. In section 3, we construct mock data of dSph member stars and foreground stars. The number of expected observed stars are estimated by assuming the model of the spectrograph and imposing the data cut. After the cut, we give a new likelihood function including the foreground contamination. The results of the fit using the new likelihood are given in section 4. Finally, we summarize our discussion in section 5 . In appendix A, we give the detailed method to estimate the foreground distribution required for the new likelihood function. We compare our results with those obtained by a conventional analysis in appendix B. Appendix $\mathrm{C}$ is devoted to show the dependence of the halo truncation radius on the $J$-factors.

\section{GAMMA-RAY FLUX FORMULA}

The differential gamma-ray flux from the dark matter annihilations in a solid angle $\Delta \Omega$ is given by

$\Phi(E, \Delta \Omega)=\left[\frac{C\langle\sigma v\rangle}{4 \pi m_{\mathrm{DM}}^{2}} \sum_{f} b_{f}\left(\frac{d N_{\gamma}}{d E}\right)_{f}\right]\left[\int_{\Delta \Omega} d \Omega \int_{\text {l.o.s. }} d l \rho^{2}(l, \Omega)\right]$.

Here $C$ is $1 / 2$ for Majorana and $1 / 4$ for Dirac dark matter, $m_{\mathrm{DM}}$ denotes the mass of the dark matter, and $\langle\sigma v\rangle$ represents the product of the total annihilation cross section 
and the relative velocity $v$ that is averaged with the velocity distribution function. The branching fraction of the annihilation into a final-state $f$ is denoted by $b_{f}$, and $\left(d N_{\gamma} / d E\right)_{f}$ is the differential number density of photons for a given final state $f$. The dark matter profile inside dSph is defined by $\rho(l, \Omega)$. The integration of the profile is done along the line-of-sight in the region of interest (ROI) $\Delta \Omega$.

We here note the uncertainties coming from the first parenthesis in the right-hand side of Eq.(1). This part is determined only by particle physics. Usually, the (velocityaveraged) total cross section and branching fraction can be obtained by a perturbative calculation and the error can be reduced to $\lesssim O(1)$ percent by a higher order calculation. The fragmentation function $\left(d N_{\gamma} / d E\right)_{f}$ usually gives larger errors. For examples, the annihilation products might be fragmented into various hadrons, ${ }^{3}$ which eventually produces stable particles ( $\operatorname{such}$ as $p, \bar{p}, e^{ \pm}, \gamma$, and $v$ ). The energy distribution of the photons is calculated by a Monte Carlo simulation such as Pythia (Sjostrand, Mrenna \& Skands 2008) or HERWIG (Corcella et al. 2001), which includes the effect of QED and QCD final-state radiations. The errors become large in the region of small photon energy (especially for $\tau^{+} \tau^{-}$channel) (Cirelli et al. 2011; Cembranos et al. 2013). However, in most cases, the uncertainty is $\lesssim 10 \%$ as long as the gamma-ray is hard enough (above $\sim 0.1 \%$ of the dark matter mass). Because the current gamma-ray detectors are sensitive for $O(0.1-1000) \mathrm{GeV}$ gamma-ray, the uncertainties in the particle factor do not significantly alter the sensitivities for the $\boldsymbol{O}(1) \mathrm{TeV}$ dark matter. Below, we discuss more important uncertainties of the astrophysical factor in the second parenthesis, where the error can reach two orders of magnitude.

\subsection{Astrophysical factor}

The second parenthesis in the right-hand side of Eq. (1), so called the $J$-factor, represents the amount of the dark matter in the halo deduced from astrophysical observation. Using the kinematical data obtained from the spectroscopic surveys, many studies provide the estimations of the $J$-factors. In Martinez (2015), the dark matter profile is evaluated by using the Bayesian hierarchical modelling where they fit the halo parameter by assuming empirical relations between the maximum velocity, maximum radius, and total luminosity with several free parameters. The fit is performed by imposing these relations as priors, while the free parameters of the relations are simultaneously optimized by utilizing all the data of multiple dwarf galaxies. On the other hand, one can also directly estimate the dark matter halo by comparing the stellar velocity data with the theoretical dispersion curve. ${ }^{4}$ The dispersion curve is obtained from the Jeans equation (Binney \& Tremaine 2008) under the assumption of spherical symmetry and steady (and dark matter domi-

3 The annihilation can also produce monochromatic gamma-rays directly via, for example, $Z \gamma, \gamma \gamma$ channel.

4 Here we note that a spectrograph can only measure stellar velocities along the line-of-sight, and they cannot be directly used for the halo estimation. nated) system, which is expressed as

$$
\frac{1}{v_{*}(r)} \frac{\partial}{\partial r} v_{*}(r) \sigma_{r}^{2}(r)+\frac{2 \beta_{\mathrm{ani}}(r) \sigma_{r}^{2}(r)}{r}=-\frac{G M(r)}{r^{2}},
$$

where $r$ denotes the distance from the centre of the dSph and $v_{*}(r)$ is the number distribution of the dSph member stars obtained from photometric observations. The velocity dispersions of the stars in the $\mathrm{dSph}$ are defined by $\sigma_{r}$, $\sigma_{\theta}$, and $\sigma_{\phi}$, which denote the components along the radial, azimuthal, and polar direction respectively. Here, for the spherical symmetry, we take $\sigma_{\theta}=\sigma_{\phi}$. The anisotropy parameter $\beta_{\text {ani }}$ is defined by $\beta_{\text {ani }}=1-\sigma_{\theta}^{2} / \sigma_{r}^{2}, G$ is the gravitational constant, and $M(r)$ is the enclosed mass of the dark matter halo.

To compare this velocity dispersion with the observables, one should project it along the line-of-sight. A straightforward calculation gives the projected dispersion curve $\sigma_{\text {l.o.s }}$ :

$$
\sigma_{\text {l.o.s }}^{2}(R)=\frac{2}{\Sigma_{*}(R)} \int_{R}^{\infty} d r\left(1-\beta_{\text {ani }}(r) \frac{R^{2}}{r^{2}}\right) \frac{\nu_{*}(r) \sigma_{r}^{2}(r)}{\sqrt{1-R^{2} / r^{2}}},
$$

where $R$ denotes the projected distance from the centre of the dSph and $\Sigma_{*}(R)$ is the projected stellar distribution obtained by integrating $v_{*}(r)$ along the projected direction. The fit is performed by comparing the observed $\sigma_{l . o . s}$ and the right-hand values of equation (3) calculated with several fitting parameters (typically the halo profile parameter and velocity anisotropy), with respect to $R$.

Recently, however, it is pointed out that there exist nonnegligible systematic errors hidden in the halo estimation: the prior biases, velocity anisotropy, halo truncation, nonsphericity, and foreground contamination.

The prior biases are required for the fit with small observational data. When the number of the observed stars is small ( $\lesssim O(100))$, the $J$-factor obtained from the fit does not converge well and gives a large uncertainty (by two orders of magnitude or more) (Bonnivard, Maurin \& Walker 2016). Therefore, most studies evaluate the $J$-factor by imposing prior biases (Martinez 2015). However, Martinez et al. (2009) reveals that the choice of the prior strongly affects the halo estimation by at least two orders of magnitude for ultrafaint dSph fit. ${ }^{5}$ Thus, conservatively speaking, one should be careful when considering the gamma-ray sensitivity lines including the contribution from the ultra-faint dSph. The effect of the prior biases becomes small when the number of the observed stars is $\sim O(1000)$, and therefore a future stellar observation of the ultra-faint dSphs is essential.

The velocity anisotropy $\beta_{\mathrm{ani}}(r)$ is another subject of the discussion (Ullio \& Valli 2016). Because the anisotropy parameter cannot be directly addressed, one should make assumptions on the spatial dependence of the anisotropy. Currently, most studies assume that the anisotropy is $r$-independent, while the recent study (Bonnivard et al. 2015b) fits the kinematical data by using the Baes \& van Hase parametrization (Baes \& Van Hese 2007). Since it is pointed out that the anisotropy parameter might give a non-negligible effect on the $J$-factor estimation (Ullio \& Valli

5 Another approach is to impose the empirical parameter cut (Bonnivard et al. 2015b). However, the physical interpretation of this cut is still unclear. 
2016), more quantitative discussion should be given in the future.

A further systematic error comes from the morphology of the outer halo. Even for the classical dSphs with $\sim 500$ member stars, the fit often allows quite large dark matter halo radius (even more than $100 \mathrm{kpc}$ ) with small dark matter density (Geringer-Sameth, Koushiappas \& Walker 2015) because the star kinematics does not provide the information over the outermost star. Although the dark matter halo may be truncated at some distance by the effect of the tidal stripping, there is no consensus on the truncation radius. One can calculate the tidal radius of the dSphs by assuming the Milky Way halo mass and profiles. Another conservative approach is to consider the distance of the outermost star as a lower bound of the truncation radius. Because smaller truncation radius gives smaller $J$-factor, this lower bound method always provides conservative results. The radius of the outermost star can be estimated by using the projected radius of the stars (Geringer-Sameth et al. 2015). In our analysis, we set the truncation radii based on this estimation and to eliminate the fluctuation due to the halo truncation, we fix the size of the halo truncation at $2000 \mathrm{pc}$, which is slightly larger truncation radius than those given by Geringer-Sameth et al. (2015) (1.9 kpc for Draco and 1.6 $\mathrm{kpc}$ for Ursa Minor). This estimation will become more accurate when larger stellar data are obtained in the future.

Recent studies also test the contribution from the nonsphericity. Although most calculations assume the spherical dark matter halo for simplicity, there is no reason why the dark matter halo should be completely spherical. Thanks to the recent improvement of the numerical resolution, N-body simulation can investigate the shape, orientation, and alignment of dSphs (Jing \& Suto 2002; Vera-Ciro et al. 2014). They show that the axis ratio is $\sim 0.6$ for most of the subhalos. Following this motivation, Hayashi et al. (2016) performs the axisymmetric fit to the observed stellar data. The results give $60-100 \%$ deviation for the $J$-factors from the spherical estimation. A consistent result is also derived by Bonnivard et al. (2015b), where they estimate the effect of the non-sphericity using mock stellar samples.

The uncertainties above can be improved as the number of the stars increases. Especially, the uncertainty of the ultra-faint dSphs is dominated by the statistical error (and hence the prior bias) and having more stars significantly suppresses their uncertainties. Meanwhile, the uncertainty of the classical dSphs stems from the choice of the model of the anisotropy and DM profile, which requires larger amount of data. Nevertheless, we expect that these model ambiguities will be resolved since the stellar data of the classical dSphs can be 3-4 times larger in the future due to the rich stellar population. In addition, future long-time observation will provide the proper motion which help determining the models.

Although the future spectroscopic survey toward the dSphs is highly motivated from the points of view of the dark matter detection, the foreground contamination remains problematic because the number of the foreground stars also increases with the deeper and wider surveys. The observed data always include the stars belonging to the Milky Way galaxy. Among them, dSph member stars are identified or weighted by membership probability (Walker et al. 2009a) by utilizing the star information such as its position, velocity, colour, metallicity, effective temperature and surface gravity. However, it is still difficult to absolutely eliminate the foreground contamination. For instance, Bonnivard et al. (2016) reveals that the profile estimation of the ultra-faint $\mathrm{dSph}$ (SegueI) is significantly affected by the 'marginal' stars, which cannot be clearly identified as member star. In the Segue I case, the overestimation of the $J$-factor reaches more than two orders of magnitude. The study also shows that the overestimation generally occurs when the number of the observed stars is $\lesssim O(100)$. Moreover, in the membership probability calculation, velocity dispersion of the member stars is assumed to be constant for its position. The velocity dispersion of dSph usually changes by factor $1.5-2$ for its position and therefore this constant assumption might become a non-negligible bias for the membership selection process and subsequent dark matter profile estimation. The halo estimation including both the foreground contamination and the position dependence of the radial velocity dispersion is required for the detailed kinematical analysis of dSphs.

The next section is devoted to investigate the potential of the future spectroscopic survey as well as the reduction of this foreground effect by introducing a new likelihood.

\section{ANALYSIS}

In the analysis, we first generate realistic mock dSph stellar data including the foreground stars. To clarify the effect of the contamination, the mock dSph stars are generated assuming the spherical distribution and a constant velocity anisotropy. Using this mock data, we test the capability of the future spectrograph and discuss the efficient data cuts. Finally, we propose a new likelihood function to eliminate the foreground bias efficiently and fit the mock data using the likelihood function. Here, we note that the fit is performed under the same assumption above (spherical, constant anisotropy).

\subsection{Dark Matter halo and stellar distribution}

In this paper, we adopt the generalized dark matter halo density profile (Hernquist 1990; Dehnen 1993; Zhao 1996) as the input dark matter profile for the mock data and fit:

$$
\rho_{\mathrm{DM}}(r)=\rho_{S}\left(r / r_{S}\right)^{-\gamma}\left(1+\left(r / r_{s}\right)^{\alpha}\right)^{-(\beta-\gamma) / \alpha},
$$

where $r$ denotes the distance from the centre of the $\mathrm{dSph}$, and parameters $\rho_{s}, r_{s}$ represent the typical density and scale of the halo respectively, while parameters $\alpha, \beta, \gamma$ determine the shape of the halo density profile. For instance, $(\alpha, \beta, \gamma)=(1,3,1)$ represents the NFW profile (Navarro, Frenk \& White 1997) and $(\alpha, \beta, \gamma) \sim(1.5,3,0)$ is the Burkert profile (Burkert 1996; Salucci \& Burkert 2000). We also assume Plummer profile (Plummer 1911) for the member stellar distribution:

$$
v_{*}(r)=\left(3 / 4 \pi r_{e}^{3}\right)\left(1+\left(r / r_{e}\right)^{2}\right)^{-5 / 2} .
$$

Here $r_{e}$ denotes the projected half-light radius of the dSph. We normalize $v_{*}(r)$ to $\int 4 \pi r^{2} v_{*}(r) d r=1$. 


\begin{tabular}{ccccc}
$\mathrm{dSph}$ & $d[\mathrm{kpc}]$ & $r_{e}[\mathrm{pc}]$ & $(l, b)$ & $v_{\mathrm{dSph}}[\mathrm{km} / \mathrm{s}]$ \\
\hline Draco & 76 & 221 & $(86.4,34.7)$ & -292 \\
Ursa Minor & 76 & 181 & $(105,44.8)$ & -247 \\
\hline
\end{tabular}

Table 1. The properties of the galaxies which are given in McConnachie (2012a) and references therein. The distances to the $d S p h$ are denoted by $d$ and $(l, b)$ are the galactic longitude and latitude of each dSph. The bulk line-of-sight velocity of each dSph is given by $v_{d S p h}$ (Falco et al. 1999; Young 2000). The negative sign represents that the $d S p h$ is moving toward the sun.

\subsection{Mock dSphs}

We construct the mock stellar data based on the classical dSphs data (where the number of the observed member stars is 300-500) with a large $J$-factor. ${ }^{6}$ This amount of the stellar data provides relatively well-determined dark matter profile and therefore, they are especially important when one considers the conservative sensitivity lines. Here we consider Draco and Ursa Minor dSphs since they have the largest $J$ factors among the classical dSphs and primary targets of the future spectrographs. Here, we extract the halo data from Draco and Ursa Minor observations and use it for the input of the mock generator (see Table 1 for the properties of each dSph).

The mock stellar data of the dSphs is constructed by two steps. At the first step, we generate the member stars with the colour and chemical information by using a stellar evolution model deduced from the current photometric and spectroscopic data. The next step is to assign to each star the information about the position and velocity consistent with the phase-space distribution function determined by the input dark matter potential.

In the first step, the synthetic colour-magnitude diagrams for the Draco and Ursa Minor are generated by utilizing the latest version of the PERSEC isochrones (Bressan et al. 2012). We first randomly draw initial masses of stars from the Salpeter initial-mass function (Salpeter 1955). Ages and metallicities are also randomly drawn from assumed distributions. The ages of the stars are assumed to be randomly distributed in the range 12.6-13.2 Gyrs for both galaxies. The stellar metallicities $([\mathrm{Fe} / \mathrm{H}])$ are assumed to follow a Gaussian distribution with a mean and dispersion $-1.9(-2.1)$ and $0.5(0.5)$ dex, respectively, for Draco (Ursa Minor), which approximately reproduce the observed metallicity distributions in these galaxies (Kirby et al. 2011). The present-day absolute magnitude, temperature, and surface gravity are then assigned to each star based on the PERSEC isochrones for the given initial mass, age, and metallicity. The apparent magnitudes are ob-

6 We also stress the importance of the ultra-faint dSphs which often have larger $J$-factors than classical dSphs. The current limited stellar data does not provide a solid value of the $\boldsymbol{J}$-factor and therefore the future spectrographic survey and our analysis for the foreground stars should play essential roles. However, other systematic errors may also largely contribute to the dark matter halo estimation and in this paper, in order to emphasize the effect of the foreground contamination, we only consider the classical case. The analysis of the ultra-faint dSphs will be investigated in our forthcoming paper. tained by taking into account the distance modulus of 19.40 , which corresponds to the distance of $76 \mathrm{kpc}$, for both Draco and Ursa Minor (McConnachie 2012b). Finally, the photometric errors, which are assumed to increase toward fainter magnitudes with a cubic polynomial, are assigned to the apparent magnitudes. The number of stars in each galaxy is adjusted to yield the total luminosity approximately consistent with the observed luminosity.

After constructing the member star mock, we next randomly assign the position and velocity of each star by using the kinematical distribution of the dSph (and finally add the bulk velocity $\left.v_{\mathrm{dSph}}\right)$. The stellar distribution consistent with the dark matter potential is obtained by the method in Cuddeford (1991) in which we assume the constant velocity anisotropy parameter. ${ }^{7}$ As the input dark matter halo, we adopt two types of the halo profile considering 'Draco-like' and 'Ursa Minor-like' dSph. For Dracolike dSphs, we estimate the halo profile by utilizing the current kinematical stellar data given by MMT/Hectochelle observations (Walker, Olszewski \& Mateo 2015). We obtain the best fit parameters of the halo by the same method in Geringer-Sameth et al. (2015) as shown in the first line in Table 2 ('Draco 1'). ${ }^{8}$ However, the best fit data for Draco usually gives large $r_{s}(\sim 10 \mathrm{kpc})$. In fact, the amount of the foreground contamination is not obvious for the current observational data. As one can see later, foreground contamination gives a large velocity dispersion at outer region and leads overestimation of the halo size. Therefore, we also adopt another fit parameter with smaller $r_{s}$ and a good chisquare $(0.1 \%$ larger than the best fit chi-square) as shown in the second line of Table 2 ('Draco 2'). For Ursa Minor-like dSphs, on the other hand, because the kinematical data of the Ursa Minor is not available, we adopt the median values of Ref(Geringer-Sameth et al. 2015) as the input parameter of the halo which is shown in the third line of Table 2 ('Ursa Minor').

The non-member stars belonging to the Milky Way galaxy are also included in the mock data. These foreground stars are generated from the Besançon model (Robin et al. 2003). The generator provides the stellar population of the Milky Way galaxy including the thin disc, thick disc, bulge and halo component with its velocity, age, luminosity, colour, chemical components, effective temperature and surface gravity. Using the Besançon model, we generate the foreground stars with spatially uniform distribution in the region of interest.

\subsection{Spectrograph}

As for the future spectrograph, we consider the Prime Focus Spectrograph (PFS) mounted on the $8.2 \mathrm{~m}$ Subaru telescope. PFS is the next generation spectrograph of the SuMiRe project (Takada et al. 2014; Sugai et al. 2015; Tamura et al.

7 In order to avoid to make unphysical distribution, we have slightly modified the input stellar distribution form by introducing the small parameter $\gamma_{*}: \gamma_{*}(r) \rightarrow\left(3-\gamma_{*}\right) / 4 \pi r_{e}^{3}\left(r / r_{e}\right)^{-\gamma_{*}}(1+$ $\left.r^{2} / r_{e}^{2}\right)^{-\left(5-\gamma_{*}\right) / 2}$. In the analysis, we have set $\gamma_{*}=0.1$ and confirmed that the fluctuations of the reproduced number distribution and dispersion curves are negligible.

8 The fit uses the $\sim 450$ kinematical data. 


\begin{tabular}{ccccccc}
$\mathrm{dSph}$ & $\log _{10}\left(\rho_{s} /\left[M_{\odot} / \mathrm{pc}^{3}\right]\right)$ & $\log _{10}\left(r_{s} /[\mathrm{pc}]\right)$ & $\alpha$ & $\beta$ & $\gamma$ & $\log _{10}\left(1-\beta_{\text {ani }}\right)$ \\
\hline Draco 1 & -2.05 & 3.96 & 2.78 & 7.78 & 0.675 & 0.130 \\
Draco 2 & -1.52 & 3.15 & 2.77 & 3.18 & 0.783 & -0.005 \\
Ursa Minor & -0.497 & 2.60 & 1.64 & 5.29 & 0.777 & -0.475 \\
\hline
\end{tabular}

Table 2. The dark matter halo parameter of each $d S p h$.

\begin{tabular}{cccccc}
$\theta_{\text {ROI }}[$ degree $]$ & $i_{\max }[\mathrm{mag}]$ & $d v[\mathrm{~km} / \mathrm{s}]$ & $d[\mathrm{Fe} / \mathrm{H}]$ & $d \log _{10}\left(g /\left[\mathrm{cm} / \mathrm{s}^{2}\right]\right)$ & $d T_{\text {eff }}[\mathrm{K}]$ \\
\hline $0.65,1.3$ & $19.5,21,21.5$ & 3.0 & 0.5 & 0.5 & 500 \\
\hline
\end{tabular}

Table 3. The capability of the spectrograph. $\theta_{R O I}$ is the radius of the region of interest.

2016), which has a large field-of-view ( 0.65 degrees $)$ and 2394 fibres. The project has a survey plan for the classical dSphs (Fornax, Sculptor, Draco, Ursa Minor, and Sextans) and aims to start the science operation from 2019-2020. The spectrograph has the three-colour arms which cover blue, red and near infrared wavelengths with the resolution $\lambda / \delta \lambda$ of 2500, 3200, and 4500, respectively. Moreover, the spectrograph has the medium-resolution option for the red-arm $(\lambda / \delta \lambda=5000)$, where the velocity precision $d v$ of $3 \mathrm{~km} / \mathrm{s}$ is expected. ${ }^{9}$ In addition, utilizing the absorption line spectrum in the wide wavelength coverage, it is expected that the detailed stellar parameters (e.g. effective temperature, surface gravity, metallicity) can be obtained with high accuracies. In the survey plan, the star with $i>21$ will be observed with an integration time of a few nights, while the current observation measures stars above $i \sim 19.5$ spending several nights to cover the dSph region.

We note that other future spectrographs planned to start operation in the next several years, such as MOONS at VLT (Cirasuolo et al. 2012), WEAVE at $4.2 \mathrm{~m}$ William Herschel Telescope (Balcells et al. 2010), DESI at 4 m Mayall telescope (Levi et al. 2013), or 4MOST at VISTA telescope (de Jong et al. 2012), could also provide velocity measurements with similar quality $(d v \leq 3 \mathrm{~km} / \mathrm{s})$. Compared to these instruments, the Subaru/PFS have both a wide field-of-view and the large aperture telescope and thus is particularly suitable for measuring velocities in the outer region of the classical dSphs down to a fainter magnitude $(i<21.0)$. Nevertheless, the analyses presented below provide a benchmark for improvement/limitation of stellar kinematic data taken with these instruments as well as with a next-generation instrument such as the Maunakea Spectroscopic Explore (MSE; Szeto et al. (2016)).

For the region of interest, we assume one-pointing observation (a radius of 0.65 degrees) and four pointing observation (1.3 degrees). We also assume that the spectrograph can measure the recession velocity $v$, metallicity $[\mathrm{Fe} / \mathrm{H}]$, effective temperature $T_{\text {eff }}$, and surface gravity $g$ for each star with the accuracies given in Table 3 , which are used to eliminate the foreground stars. ${ }^{10}$ To simulate the detector resolution, the mock data is smeared by the normal distribution func-

9 The medium resolution mode $(\lambda=7100-8850 \AA)$ covers the Calcium triplet and $\alpha$ element lines.

10 We check the effect of the velocity resolution and find it small because the dispersion is given by $\sqrt{\sigma_{r}^{2}+d v^{2}}$. For classical dSph tions with the respective resolution widths given in Table 3 . As the depth of the survey depends on the exposure time, we adopt three cases of the upper bound of the magnitude $\left(i_{\max }=19.5,21,21.5\right)$. In the first case, we demonstrate the current sensitivity reach and the effect of the foreground contamination. The maximum magnitude $i_{\max }=19.5$ is chosen to reproduce the current number of stellar data $(\sim 300)$. The second case $\left(i_{\max }=21\right)$ corresponds to the expected reach of PFS one-pointing observation with an integration time of a few nights. As for the multi-pointing case, we consider the option with a wider region of interest $\left(\theta_{\mathrm{ROI}}=1.3\right.$ degree $)$. The final case $\left(i_{\max }=21.5\right)$ is for a deeper survey with an integration time of several nights, in which we consider only the one-pointing case.

\subsection{Cut}

The mock data obtained in Sec. 3.2 contain a large number of the foreground stars. To reduce the foreground contamination, we impose cuts on the raw mock data. ${ }^{11}$ First, the spatial $\left(r<d \sin \theta_{\mathrm{ROI}}\right)$ cut should be imposed because of the limited region of interest. Here $d$ denotes the distance of each dSph and $\theta_{\mathrm{ROI}}$ is the angular radius of the region of interest. We further optimize the foreground contamination by imposing following cuts:

- $v_{\text {lower }}<v<v_{\text {upper }}$,

- $0.2<\log _{10}\left(\mathrm{~g} /\left[\mathrm{cm} / \mathrm{s}^{2}\right]\right)<3.7$,

as well as the cut on the colour-magnitude diagram as shown in Fig. 1. Here, $v_{\text {lower }}, v_{\text {upper }}$ are given in Table 4 for each dSph. We choose the velocity bound to include most of stars in clumps (by eyes). A harder cut can be imposed to reduce the fraction of the foreground stars. However, in that case, since the scattered member stars are also eliminated, the reconstructed velocity distribution may be biased. The effect of this hard cut is beyond the scope of this paper and we do not go into further details. The number of the

case, $\sigma_{r} \gtrsim 10(\mathrm{~km} / \mathrm{s})$ and the effect of $d v$ negligibly contributes to the $J$-factor uncertainty.

11 Standard analysis of $J$-factor estimation uses stars selected according to their membership probabilities, which can be a more sophisticated selection than the cut method introduced in this section. The distribution of the selected data can be different from that obtained by the cut. Therefore it is worth comparing the results with those given by the standard analysis. We discuss it in appendix B. 


\begin{tabular}{|c|c|c|c|c|c|c|}
\hline dSph & $\theta_{\mathrm{ROI}}[$ degree] & $i_{\max }[\mathrm{mag}]$ & $v_{\text {lower }}[\mathrm{km} / \mathrm{s}]$ & $v_{\text {upper }}[\mathrm{km} / \mathrm{s}]$ & $N_{\mathrm{Mem}}$ & $N_{\mathrm{FG}}$ \\
\hline \multirow[t]{4}{*}{ Draco $1 \& 2$} & 0.65 & 19.5 & -350 & -230 & 260 & 16 \\
\hline & & 21 & & & 900 & 37 \\
\hline & & 21.5 & & & 1140 & 43 \\
\hline & 1.3 & 21 & & & 940 & 150 \\
\hline \multirow[t]{4}{*}{ Ursa Minor } & 0.65 & 19.5 & -310 & -190 & 290 & 10 \\
\hline & & 21 & & & 1100 & 33 \\
\hline & & 21.5 & & & 1400 & 41 \\
\hline & 1.3 & 21 & & & 1130 & 140 \\
\hline
\end{tabular}

Table 4. The status of the mock dSphs. The averaged number of the member (foreground) stars after the cuts are given by $N_{M e m}\left(N_{F G)}\right.$. See the text for the details of the cuts.
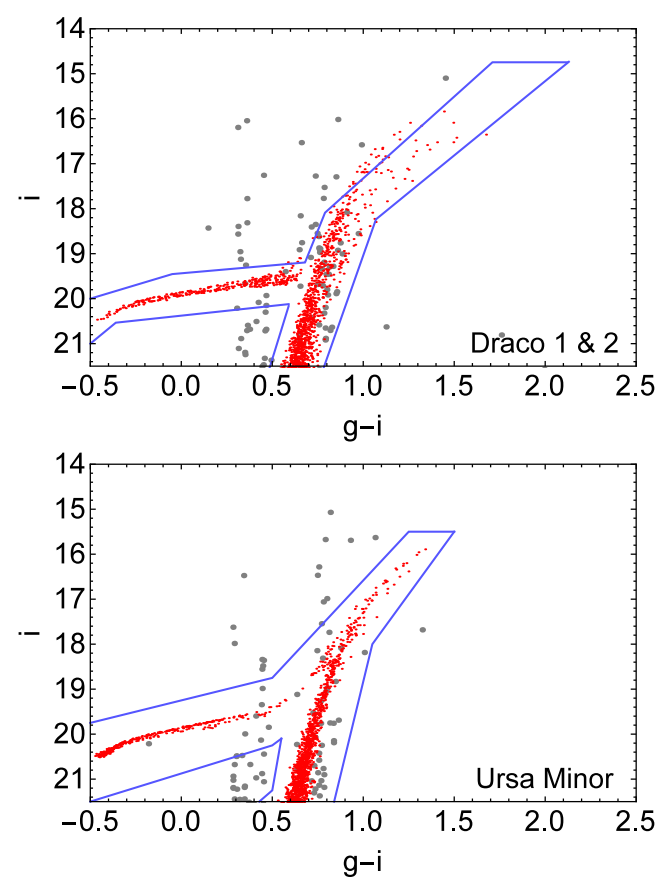

Figure 1. The colour-magnitude map for Draco (top) and Ursa Minor (bottom). We impose the colour-magnitude cut by the blue lines. The red (grey) dots show the members (foreground) stars. The stars on the map are residuals after the cuts of the ROI, velocity, and $\log g$. For Draco, we draw the colour cut referring Walker et al. (2015).

member and foreground stars after imposing the cuts is also shown in the table. Since the bulk velocity of the dSphs is largely different from the Milky Way stellar halo component (see Fig.A1 in appendix A), the velocity cut works most efficiently. We also note that because the surface gravity reflects the absolute magnitude, the surface gravity cut can efficiently eliminate the faint foreground stars from the member stars with brighter absolute magnitude. As most of the residual foreground stars belong to the Milky Way stellar halo component, additional cuts using the metallicity or effective temperature are not efficient because the member stars are indistinguishable from the halo stars in terms of these quantities for the accuracy given in Table 3. Rather, these additional cuts eliminate the member stars scattered by the detector resolution by $15 \%$ level.

\subsection{Velocity dispersion}

The velocity dispersion along the line-of-sight can be obtained from Eq.(2)and (3). Here we note that Eq.(2) has a general solution (van der Marel 1994; Mamon \& Eokas 2005). When the $\beta_{\text {ani }}$ is constant, the radial velocity dispersion can be expressed as

$$
\sigma_{r}^{2}(r)=\frac{1}{v_{*}(r)} \int_{r}^{\infty} v_{*}\left(r^{\prime}\right)\left(\frac{r^{\prime}}{r}\right)^{2 \beta_{\text {ani }}} \frac{G M\left(r^{\prime}\right)}{r^{\prime 2}} d r^{\prime} .
$$

Combining Eq.(3)-(6), we convert $\sigma_{r}^{2}(r)$ to the projected velocity dispersion $\sigma_{\text {l.o.s }}^{2}(R)$, which is calculated by inputting halo parameters $\alpha, \beta, \gamma, \rho_{s}, r_{s}$ and velocity anisotropy $\beta_{\text {ani }}$. We also note that $M(r) \equiv \int_{0}^{r} 4 \pi r^{\prime 2} \rho\left(r^{\prime}\right) d r^{\prime}$ under the spherical assumption and we define $\Sigma_{*}(R)=\left(1 / \pi r_{e}^{2}\right)\left(1+\left(R / r_{e}\right)^{2}\right)^{-2}$ for the Plummer profile.

\subsection{Likelihood}

The likelihood function is constructed taking the foreground contamination into account. To avoid the binning dependence, we perform the unbinned analysis by setting the likelihood as follows:

$$
-2 \ln \mathcal{L}=-2 \sum_{i} \ln \left(s f_{\mathrm{Mem}}\left(v_{i}, R_{i}\right)+(1-s) f_{\mathrm{FG}}\left(v_{i}, R_{i}\right)\right),
$$

where $s$ is the membership fraction parameter and $f_{\mathrm{Mem}}(v, R)\left(f_{\mathrm{FG}}(v, R)\right)$ is the distribution function of the member (foreground) stars. The index $i$ runs all the stars in the mock data set. The distribution functions are defined by

$$
\begin{aligned}
& f_{\mathrm{Mem}}(v, R)=2 \pi R \Sigma_{*}(R) C_{\mathrm{Mem}} \mathcal{G}\left[v ; v_{\mathrm{Mem}}, \sigma_{l . o . s}(R)\right], \\
& f_{\mathrm{FG}}(v, R)=2 \pi R C_{\mathrm{FG}} \mathcal{G}\left[v ; v_{\mathrm{FG}}, \sigma_{\mathrm{FG}}\right],
\end{aligned}
$$

where $\sigma_{\mathrm{FG}}$ does not depend on $R$. Here, we assume that both of the velocity distributions can be approximated by a single Gaussian, and hence, $\mathcal{G}[x ; \mu, \sigma]$ denotes the Gaussian distribution of a variable $x$ with a mean value, $\mu$ and a standard deviation, $\sigma$. The parameter $v_{\text {Mem }}$ represents the bulk velocity of the dSph while $v_{\mathrm{FG}}$ is (dominantly) controlled by the bulk velocity of the foreground halo component, which are treated as nuisance parameters in the following analysis. $C_{\mathrm{Mem}}$ and $C_{\mathrm{FG}}$ are the normalization correction factor, 
under which the distribution functions satisfy

$$
\begin{gathered}
\int_{0}^{r_{\mathrm{ROI}}} d R \int_{v_{\text {lower }}}^{v_{\text {upper }}} d v f_{\mathrm{Mem}}(v, R)=1, \\
\int_{0}^{r_{\mathrm{ROI}}} d R \int_{v_{\text {lower }}}^{v_{\text {upper }}} d v f_{\mathrm{FG}}(v, R)=1,
\end{gathered}
$$

where $r_{\text {ROI }} \equiv d \sin \theta_{\text {ROI }}$. We note that the free parameter $v_{\text {Mem }}$ always converges to the input bulk velocity $v_{\mathrm{dSph}}$.

Before the fit, the information about $v_{\mathrm{FG}}, \sigma_{\mathrm{FG}}$ can be extracted by utilizing the data set in the control region, i.e., the data set with $v<v_{\text {lower }}$ or $v>v_{\text {upper. }}{ }^{12}$ Performing a fit to the control region, the best fit value and standard deviation of $v_{\mathrm{FG}}, \sigma_{\mathrm{FG}}\left(v_{\mathrm{FG} 0}, \sigma_{\mathrm{FG} 0}, d v_{\mathrm{FG}}, d \sigma_{\mathrm{FG}}\right)$ can be obtained. We use this information as a prior for $v_{\mathrm{FG}}, \sigma_{\mathrm{FG}}$ by multiplying $\mathcal{G}\left[v_{\mathrm{FG}} ; v_{\mathrm{FG} 0}, d v_{\mathrm{FG}}\right] \mathcal{G}\left[\sigma_{\mathrm{FG}} ; \sigma_{\mathrm{FG} 0}, d \sigma_{\mathrm{FG}}\right]$ to the likelihood function $\mathcal{L}$ in Eq.(7). Here we emphasize that to construct the foreground distribution function, the amount of the foreground data is essential. For instance, the number of the stars after the colour and ROI cuts is $\sim 2000$ for the one-pointing case. Such a large number of stars can only be accessed by the spectrograph with many fibres, like PFS. The detailed method to estimate the foreground distribution function is given in appendix A. ${ }^{13}$ We check that the parameters of the foreground distribution $\left(v_{\mathrm{FG}}, \sigma_{\mathrm{FG}}\right)$ converges within $\sim 1$ sigma width of the prior on average.

The Bayesian posterior probability function of the five free parameters of the dark matter halo $\left(\rho_{s}, r_{s}, \alpha, \beta, \gamma\right)$, one velocity anisotropy parameter $\beta_{\text {ani }}$ and four nuisance parameters in the likelihood function $\left(s, v_{\mathrm{Mem}}, v_{\mathrm{FG}}, \sigma_{\mathrm{FG}}\right)$ is obtained from the likelihood function in Eq.(7) multiplied by the foreground priors. We perform the MetropolisHastings (MH) algorithm (Metropolis et al. 1953; Hastings 1970) of the Markov Chain Monte Carlo (MCMC) method. Once one properly tunes the MCMC process (such as the number of the burn-in step, the sampling step, and length of the chain), the sampling set of the MCMC reflects the probability density of the likelihood function. Accumulating $O\left(10^{5}\right)$ samples for each data set, we search the halo parameters under the flat/log-flat priors within the range of $-4<\log _{10}\left(\rho_{s} /\left[\mathrm{M}_{\odot} / \mathrm{pc}^{3}\right]\right)<4,0<\log _{10}\left(r_{s} /[\mathrm{kpc}]\right)<5,0.5<$ $\alpha<3,3<\beta<10,0<\gamma<1.2$ and $-1<\log _{10}\left(1-\beta_{\text {ani }}\right)<1$, which are the same criteria of Geringer-Sameth et al. (2015).

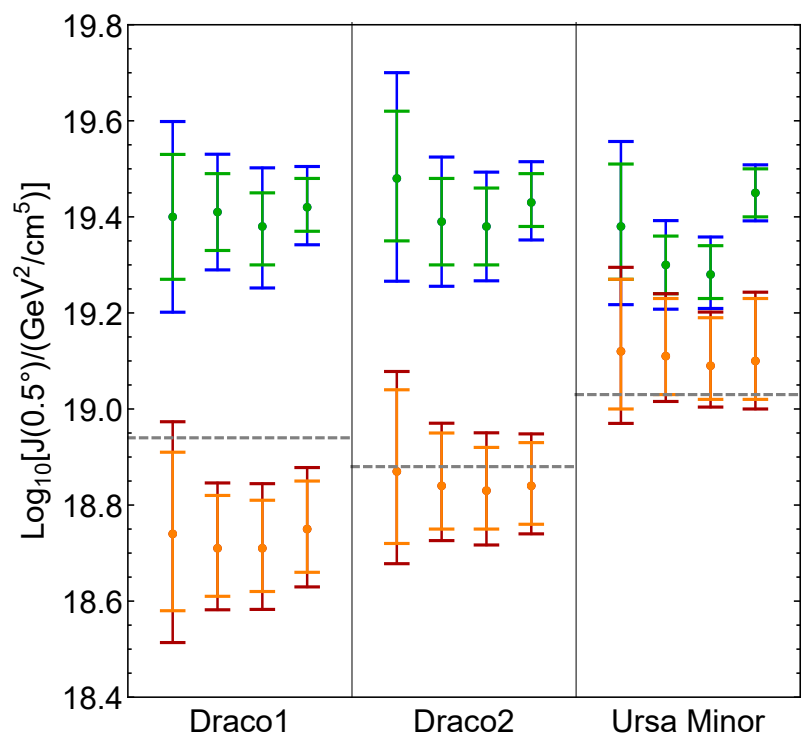

Figure 2. The $J$-factors in Table 5 are plotted. The orange dots show the $J$-factor estimation of 'Our fit', while the green dots represent the 'Contaminated' results. The orange and green bars are the first uncertainties in Table 5. The red and blue bars are the errors where the standard deviation of the median values (the second uncertainties in Table 5) is added by square root sum. The grey dashed lines show the input values. For each $d S p h$, the first three bars from the left correspond to the case of $i_{\max }=$ 19.5, 21, 21.5 with $\theta_{R O I}=0.65$ and the last bar is $i_{\max }=21$ with $\theta_{\text {ROI }}=1.3$ in Table 5

\section{RESULTS}

Table 5 and Fig. 2 show the results of the fit. We produce 50 mocks for each case and average the median values of $\log _{10}\left(J /\left[\mathrm{GeV}^{2} / \mathrm{cm}^{5}\right]\right)$. The error bar of the $J$-factor for each mock sample is estimated by comparing the median and $68 \%$ quantile. In the table, we give the averages of the error bars at the first uncertainties. The standard deviation of the median values is also put on the second uncertainty, which reflects the statistical deviation of the sample quality. All $J$-factors are calculated in $\Delta \Omega=2.4 \times 10^{-4} \mathrm{sr}$, corresponding to the angular radius of 0.5 degree, which is the standard size for the $J$-factor calculation (Ackermann et al. 2015). ${ }^{14}$ To eliminate the fluctuation due to the halo trun-

12 The control region can also be taken by the spatial position, setting an annulus centred at the dSph galaxy. However, we have found that the fraction of the member star in the annulus is not negligible and therefore we decided to use $v$ to define the control region.

13 We here note that this fit procedure is essentially the same as the one proposed in the appendix of Bonnivard et al. (2016) as an alternative procedure for their main analysis. However, the main difference is that we derive the foreground distribution function by utilizing the control region, which is introduced as the prior function in the likelihood. As another difference, we use the information about the metallicity only for the cut and do not include them in the likelihood. This is because the metallicities of the member stars and foreground halo components are highly degenerate with each other.

14 We also check how the error of the $J$-factor are affected by changing this integration angle. For Draco 1, Draco 2, and 


\begin{tabular}{cccccc}
\multicolumn{2}{c}{ Condition } & \multicolumn{4}{c}{$\log _{10}\left(J /\left[\mathrm{GeV}^{2} / \mathrm{cm}^{5}\right]\right)$} \\
\hline Mock & $\theta_{\text {ROI }}$ & $i_{\max }$ & Input & Contaminated & Our fit \\
\hline \multirow{2}{*}{ Draco 1 } & 0.65 & 19.5 & 18.94 & $19.40_{-0.13}^{+0.13} \pm 0.15$ & $18.74_{-0.16}^{+0.17} \pm 0.16$ \\
& & 21 & & $19.41_{-0.08}^{+0.08} \pm 0.09$ & $18.71_{-0.10}^{+0.11} \pm 0.08$ \\
& & 21.5 & & $19.38_{-0.08}^{+0.07} \pm 0.10$ & $18.71_{-0.09}^{+0.10} \pm 0.09$ \\
& 1.3 & 21 & & $19.42_{-0.05}^{+0.06} \pm 0.06$ & $18.75_{-0.09}^{+0.10} \pm 0.08$ \\
\hline \multirow{2}{*}{ Draco 2 } & 0.65 & 19.5 & 18.88 & $19.48_{-0.13}^{+0.14} \pm 0.17$ & $18.87_{-0.15}^{+0.17} \pm 0.12$ \\
& & 21 & & $19.39_{-0.09}^{+0.09} \pm 0.10$ & $18.84_{-0.09}^{+0.11} \pm 0.07$ \\
& 1.3 & 21 & & $19.38_{-0.08}^{+0.08} \pm 0.08$ & $18.83_{-0.08}^{+0.09} \pm 0.08$ \\
& & $19.43_{-0.05}^{+0.06} \pm 0.06$ & $18.84_{-0.08}^{+0.09} \pm 0.06$ \\
\hline Ursa Minor & 0.65 & 19.5 & 19.03 & $19.38_{-0.11}^{+0.13} \pm 0.12$ & $19.12_{-0.12}^{+0.15} \pm 0.09$ \\
& & 21 & & $19.30_{-0.06}^{+0.06} \pm 0.07$ & $19.11_{-0.08}^{+0.12} \pm 0.05$ \\
& & 21.5 & & $19.28_{-0.05}^{+0.06} \pm 0.05$ & $19.09_{-0.07}^{+0.10} \pm 0.05$ \\
& 1.3 & 21 & & $19.45_{-0.05}^{+0.05} \pm 0.03$ & $19.10_{-0.08}^{+0.13} \pm 0.06$ \\
\hline
\end{tabular}

Table 5. The resultant $J$-factors calculated within an angular radius of $0.5^{\circ}$. We produce 50 mocks and we give the mean (the first values) and averages of the error bars (the first uncertainties). The standard deviation of the median values is also put on the second uncertainty. The $J$-factors calculated by the input parameters are given in the 'Input' column. The 'Contaminated' column shows the results where all the data after the cut are considered as the member. The 'Our fit' column shows the fit results obtained by using the likelihood of Eq.(7).

cation, as we have mentioned in Sec. 2.1, we fix the size of the halo truncation at 2000 pc. ${ }^{15}$ The $J$-factors calculated by the input parameters are given in the 'Input' column. The 'Contaminated' column shows the results where all the data after the cut are considered as the member (meaning that we fix $s=1$ in Eq.(7)). The 'Our fit' column shows the results obtained by using the likelihood of Eq.(7).

As one can see from the figure, the results obtained from our likelihood successfully reproduces the input value. For $i_{\max }=21$ case, the estimation error (the first uncertainty) is about $20-35$ percent smaller than $i_{\max }=19.5$ case. Although $i_{\max }=21$ observation provides $3-4$ times larger number of observed stars, the improvement of the $J$-factor estimation is rather mild. This is because the error below $\delta \log J \sim 0.1$ is dominated by the degeneracy of the parameter $r_{s}, \rho_{s}, \gamma$, and $\beta_{a n i}$, which is hard to reduce by the projected kinematical data, as one can see in Bonnivard et al. (2015a). This degeneracy will be resolved by measuring the proper motion of the stars by the future photometric longtime observations. By the same reason, the four-pointing case and $i_{\max }=21.5$ case do not significantly improve the $J$-factor estimation, although the error of the $J$-factor becomes slightly small in both cases. It implies in turn that only the one-pointing observation provides enough stellar data to construct both member and foreground distribution.

The second uncertainty in the table shows the sampling fluctuation coming from the quality of the data, which is not included in the uncertainty of the conventional kinematical

Ursa Minor case, we find that the integration angle of $\sim 0.3$ degree gives the smallest error bar for the $J$-factor estimations, which corresponds to a scale of $\sim 2 r_{e}$. A wider integration angle gives a larger error and the error bar of 0.5 degree is larger than that minimum by a factor of two at most. Therefore, more robust estimations can be derived for these dSphs by tuning the integration angles.

15 We give the effect by changing this truncation radius in appendix $\mathrm{C}$. fit. The result shows that this fluctuation is subdominant but not negligible especially for the $i_{\max }=19.5$ case, in which the median value of the $J$-factor has $O(0.1)$ fluctuation. As for the number of the samples becomes about $3-4$ times larger, the $i_{\max }=21,21.5$ cases provide about half of the sampling error of $i_{\max }=19.5$ case. The difference between $i_{\max }=21$ and $i_{\max }=21.5$ cases is not obvious since the number of the observed stars is similar.

As an example of the result, we show the dispersion curve obtained from the best fit parameter and mock data in Fig 3. Although we do not adopt the binned analysis, the fit successfully reproduces the input curves. The 'Contaminated' column, on the other hand, shows that even if the contamination is 3-10 percent (see Table 4) and the number of the observed stars is large, the fit gives significantly large $J$-factor $(\delta \log J \sim 0.3-0.5)$. This systematic error stems from the enhancement of the velocity dispersion by the foreground stars at the outer region.

Although our likelihood estimation gives consistent results with the 'Input' values, small discrepancies appear for the Draco 1 and Ursa Minor case. In these fits, the likelihood distribution has a broad and flat peak (especially along the $r_{s}$ axis) and the scattered points around the input parameter contribute to the $J$-factor distribution asymmetrically around the input $J$-factor value. As a result, the distribution of the $J$-factor is distorted from a Gaussian shape and its peak does not always coincide with the input $J$ factor value, which generates the discrepancy between the median and input $J$-factors. The tendency of this $J$-factor bias is basically determined by some combinations of the input parameters and the precise prediction is rather difficult. For example, for Ursa Minor case, the results give slightly higher $J$-factors than the input value. We have checked this tendency by using the member star only data and found that for $i_{\max }=21, \theta_{\mathrm{ROI}}=0.65$ degree, the fit gives $\log _{10}\left(\mathrm{~J} /\left[\mathrm{GeV}^{2} / \mathrm{cm}^{5}\right]\right)=19.09_{-0.08}^{+0.13} \pm 0.03$ for Ursa Minor. For the Draco 1 case, on the other hand, the fit using only the member stars gives $\log _{10}\left(\mathrm{~J} /\left[\mathrm{GeV}^{2} / \mathrm{cm}^{5}\right]\right)=18.86_{-0.08}^{+0.09} \pm 0.08$, 

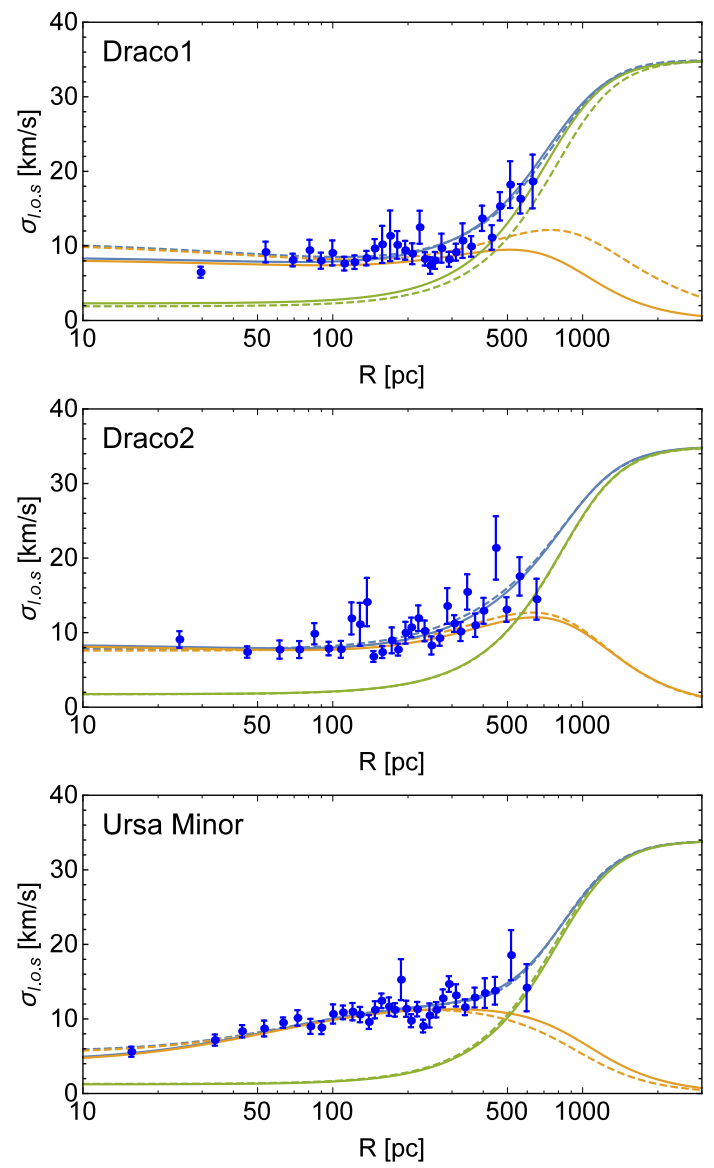

Figure 3. The dispersion curve of $\theta_{R O I}=0.65, i_{\max }=21$ case of Draco 1 (top), Draco 2 (middle), and Ursa Minor (bottom). The binned dispersions of the mock data are shown by the blue dots with error bars. The solid blue line shows the dispersion curve obtained from the best fit parameter. The solid orange (green) line shows the member (foreground) contribution to the dispersion curve. The dashed orange line is obtained from the input parameter of the dSph dark matter halo. The dashed green line is the curve obtained from the mean value of the foreground prior, while the dashed blue line shows the sum of them.

which is slightly smaller than the input $J$-factor. These results imply that even if we could perfectly reduce the foreground star, the degeneracy of the parameter gives a small discrepancy of the $J$-factors.

For the Draco 1 case, the foreground contamination gives another bias in our fit, allowing smaller $r_{s}$ region under a fixed halo radius $\rho_{s}$. This bias can be explained as follows: Consider the stellar distribution with respect to its velocity (like Fig. A1). In the region of $v_{\text {lower }}<v<v_{\text {upper }}$, the foreground velocity distribution monotonically increases as the velocity increases. On the other hand, the member stars distribute like Gaussian at $v=v_{\mathrm{dSph}}$ with a standard deviation of $\sigma_{\text {l.o.s }}(R)$. The properties of these distributions imply that more miss-identification occur in the $v>v_{\mathrm{dSph}}$ region, where the number of the foreground stars increases. Due to this asymmetric uncertainty, the fit tends to underestimate the width of the velocity distribution of the member stars. In particular, when the dark matter halo size $r_{s}$ is large like the Draco 1 case, the velocity dispersion of the mem- ber stars becomes large at the outer region $R \gtrsim O(1) \mathrm{kpc}$, in which the fraction of the foreground star increases. As a result, the identification becomes more difficult in this outer region, deriving the underestimation of the halo size as in the case of Draco 1. One should, therefore, be aware of the systematic errors when the resultant $r_{s}$ is, roughly speaking, larger than the maximum distance of the observed stars.

The same approach can be applied to the other classical dSphs. For larger dSphs such as Fornax, Sculptor, and Carina, observations with multiple-pointings may be required to cover all the member stars. However, even in that case, the total exposure time does not inflate because the number of the bright member stars are sufficiently large. Moreover, the multiple-pointings observation will also bring the information about the tidal radius of each dSph.

Before closing this section, we stress that the result of the current data (corresponding to the contaminated case of $i_{\max }=19.5$ ) indicates the existence of a non-negligible systematic bias from the foreground stars even for the classical dSph with $\mathrm{O}(100)$ stellar data. Usually, the foreground stars are eliminated by imposing the membership probability (Walker et al. 2009a), in which the stellar data with the probability above $95 \%$ is considered as a member star. However, our analysis shows that only $5 \%$ contamination significantly leads to the overestimation of the $\boldsymbol{J}$-factor by a factor of three and gives an incorrectly stringent limit to the dark matter annihilation cross section. Therefore, we conclude that a careful treatment of the foreground estimation is required to study the dark matter nature.

\section{SUMMARY}

In this paper, we have investigated the future impact of the stellar observation and the effect of the foreground contamination by using the mock dSph samples. We have tested the various cuts to optimize the quality of the data and found that the cuts on the velocity and surface gravity efficiently eliminate the contamination, while other cuts do not work well because the member stars and the halo stars degenerate. A new likelihood function has been constructed which includes the foreground distribution function. We have tested the likelihood function by making the three types of the mock data (Draco with large $r_{s}$, Draco with small $r_{s}$, and Ursa Minor) and four cases of the observation (small/large ROI, $i_{\max }=19.5,21$, and 21.5). The likelihood successfully reproduces the input $J$-factor value while the contaminated fit gives large deviation from the input value. The small discrepancy between the input $J$-factor and median value obtained by our fit suggests that the parameter degeneracy in the likelihood function distorts the $J$-factor distribution from the Gaussian shape with a peak at the input value and gives an asymmetric distribution. This non-Gaussianity may affect the estimation of the sensitivity lines of dark matter detection. We have also found that the reduction of the foreground effect becomes worse when the halo radius is large (roughly larger than the outermost observed star), which causes $\delta \log J \sim 0.2$ deviation. The effect of the sampling fluctuation has also been estimated. It is found that the statistical fluctuation of the sampling leads $\delta \log J \sim 0.1$ at most even for the fit under $O(1000)$ samples. 


\section{Acknowledgments}

This work is supported in part by a Grants-in-Aid for Scientific Research from the Japan Society for the Promotion of Science (JSPS) (No. 25-7047 for M. N. I. and No. 26-3302 for K.H.) and from the Ministry of Education, Culture, Sports, Science, and Technology (MEXT), Japan, No. 16H01090 (for K. H.), No. 15H05889, No. 25105011 (for M. I.), No. 16H02176, No. 26104009 (for S. M.), and No. 26287039 (for S. M. and M. I.). The work of K.I. is also supported by the JSPS Research Fellowships for Young Scientists. Finally, Kavli IPMU is supported by World Premier International Research Center Initiative (WPI), MEXT, Japan.

\section{REFERENCES}

Ackermann M., et al., 2015, Phys. Rev. Lett., 115, 231301

Ade P. A. R., et al., 2016, Astron. Astrophys., 594, A13

Aden D., et al., 2009, Astron. Astrophys., 506, 1147

Baes M., Van Hese E., 2007, Astron. Astrophys., 471, 419

Balcells M., et al., 2010, Proc. SPIE Int. Soc. Opt. Eng., 7735, $7 \mathrm{G}$

Bhattacherjee B., Ibe M., Ichikawa K., Matsumoto S., Nishiyama K., 2014, JHEP, 07, 080

Binney J., Tremaine S., 2008, Galactic Dynamics: Second Edition. Princeton University Press

Bonnivard V., Combet C., Maurin D., Walker M. G., 2015a, Mon. Not. Roy. Astron. Soc., 446, 3002

Bonnivard V., et al., 2015b, Mon. Not. Roy. Astron. Soc., 453, 849

Bonnivard V., Maurin D., Walker M. G., 2016, Mon. Not. Roy. Astron. Soc., 462, 223

Bradac M., et al., 2006, Astrophys. J., 652, 937

Bressan A., Marigo P., Girardi L., Salasnich B., Dal Cero C., Rubele S., Nanni A., 2012, MNRAS, 427, 127

Burkert A., 1996, IAU Symp., 171, 175

Cembranos J. A. R., de la Cruz-Dombriz A., Gammaldi V., Lineros R. A., Maroto A. L., 2013, JHEP, 09, 077

Cirasuolo M., et al., 2012, Proc. SPIE Int. Soc. Opt. Eng., 8446, $84460 \mathrm{~S}$

Cirelli M., et al., 2011, JCAP, 1103, 051

Clowe D., Bradac M., Gonzalez A. H., Markevitch M., Randall S. W., Jones C., Zaritsky D., 2006, Astrophys. J., 648, L109

Corcella G., Knowles I. G., Marchesini G., Moretti S., Odagiri K., Richardson P., Seymour M. H., Webber B. R., 2001, JHEP, 01,010

Cuddeford P., 1991, "Mon. Not. Roy. Astron. Soc.", 253, 414

Dehnen W., 1993, Mon. Not. Roy. Astron. Soc., 265, 250

Falco E. E., et al., 1999, Publ. Astron. Soc. Pac., 111, 438

Feng J. L., 2010, Ann. Rev. Astron. Astrophys., 48, 495

Geringer-Sameth A., Koushiappas S. M., Walker M., 2015, Astrophys. J., 801, 74

Hastings W. K., 1970, Biometrika, 57, 97

Hayashi K., Ichikawa K., Matsumoto S., Ibe M., Ishigaki M. N., Sugai H., 2016, Mon. Not. Roy. Astron. Soc., 461, 2914

Hernquist L., 1990, Astrophys. J., 356, 359

Ichikawa K., Ishigaki M. N., Matsumoto S., Ibe M., Sugai H., Hayashi K., Horigome S.-i., 2017, in preparation.

Jing Y. P., Suto Y., 2002, Astrophys. J., 574, 538

Jungman G., Kamionkowski M., Griest K., 1996, Phys. Rept., 267,195

Kirby E. N., Lanfranchi G. A., Simon J. D., Cohen J. G., Guhathakurta P., 2011, ApJ, 727, 78

Kirby E. N., Boylan-Kolchin M., Cohen J. G., Geha M., Bullock J. S., Kaplinghat M., 2013, Astrophys. J., 770, 16
Kirby E. N., Simon J. D., Cohen J. G., 2015, Astrophys. J., 810, 56

Koch A., Kleyna J. T., Wilkinson M. I., Grebel E. K., Gilmore G. F., Evans N. W., Wyse R. F. G., Harbeck D. R., 2007, Astron. J., 134, 566

Koposov S. E., et al., 2011, Astrophys. J., 736, 146

Levi M., et al., 2013

Lokas E. L., Mamon G. A., 2003, Mon. Not. Roy. Astron. Soc., 343,401

Mamon G. A., Łokas E. L., 2005, Mon. Not. Roy. Astron. Soc., 363, 705

Martin N. F., et al., 2016, Astrophysical Journal, 818, 40

Martinez G. D., 2015, Mon. Not. Roy. Astron. Soc., 451, 2524

Martinez G. D., Bullock J. S., Kaplinghat M., Strigari L. E., Trotta R., 2009, JCAP, 0906, 014

Mateo M., Olszewski E. W., Walker M. G., 2008, Astrophys. J., 675,201

McConnachie A. W., 2012a, Astron. J., 144, 4

McConnachie A. W., 2012b, AJ, 144, 4

McConnachie A. W., Cote P., 2010, Astrophys. J., 722, L209

McLaughlin D. E., 1999, Astrophys. J., 512, L9

Metropolis N., Rosenbluth A. W., Rosenbluth M. N., Teller A. H., Teller E., 1953, J. Chem. Phys., 21, 1087

Minor Q. E., 2013, Astrophys. J., 779, 116

Murayama H., 2007, in Les Houches Summer School - Session 86: Particle Physics and Cosmology: The Fabric of Spacetime Les Houches, France, July 31-August 25, 2006. (arXiv:0704.2276), https://inspirehep.net/record/748786/files/arXiv:0704.2276.pdf

Navarro J. F., Frenk C. S., White S. D. M., 1997, Astrophys. J., 490, 493

Olszewski E., Pryor C., Armandroff T., 1996, Astron. J., 111, 750

Plummer H. C., 1911, Mon. Not. Roy. Astron. Soc., 71, 460

Robin A. C., Reyle C., Derriere S., Picaud S., 2003, Astron. Astrophys., 409, 523

Rubin V. C., Thonnard N., Ford Jr. W. K., 1978, Astrophysical Journal, 225, L107

Rubin V. C., Ford W. K. J., . Thonnard N., 1980, Astrophysical Journal, 238, 471

Salpeter E. E., 1955, Astrophys. J., 121, 161

Salucci P., Burkert A., 2000, Astrophys. J., 537, L9

Simon J. D., Geha M., 2007, Astrophys. J., 670, 313

Simon J. D., et al., 2011, Astrophys. J., 733, 46

Simon J. D., et al., 2015a, Astrophysical Journal, 808, 95

Simon J. D., et al., 2015b, Astrophys. J., 808, 95

Sjostrand T., Mrenna S., Skands P. Z., 2008, Comput. Phys. Commun., 178, 852

Sugai H., et al., 2015, Journal of Astronomical Telescopes, Instruments, and Sy 1,035001

Szeto K., et al., 2016, Proc. SPIE, 9906, 99062J

Takada M., et al., 2014, Publ. Astron. Soc. Jap., 66, R1

Tamura N., et al., 2016, Proc. SPIE Int. Soc. Opt. Eng., 9908, $99081 \mathrm{M}$

Ullio P., Valli M., 2016, JCAP, 1607, 025

Vera-Ciro C., Sales L. V., Helmi A., Navarro J. F., 2014, Mon. Not. Roy. Astron. Soc., 439, 2863

Walker M. G., Mateo M., Olszewski E. W., Bernstein R. A., Wang X., Woodroofe M., 2006, Astron. J., 131, 2114

Walker M. G., Mateo M., Olszewski E. W., Sen B., Woodroofe M., 2009a, Astron. J., 137, 3109

Walker M. G., Belokurov V., Evans N. W., Irwin M. J., Mateo M., Olszewski E. W., Gilmore G., 2009b, Astrophys. J., 694, L144

Walker M. G., Mateo M., Olszewski E. W., Penarrubia J., Evans N. W., Gilmore G., 2009c, Astrophys. J., 704, 1274

Walker M. G., Olszewski E. W., Mateo M., 2015, Mon. Not. Roy. Astron. Soc., 448, 2717

York D. G., et al., 2000, Astron. J., 120, 1579 
Young L. M., 2000, Astron. J., 119, 188

Zhao H., 1996, Mon. Not. Roy. Astron. Soc., 278, 488

Zwicky F., 1933, Helvetica Physica Acta, 6, 110

de Jong R. S., et al., 2012, Proc. SPIE Int. Soc. Opt. Eng., 8446, $84460 \mathrm{~T}$

van der Marel R. P., 1994, Mon. Not. Roy. Astron. Soc., 270, 271

\section{APPENDIX A: FOREGROUND DISTRIBUTION}

In this appendix, we mention the method to obtain the foreground prior discussed in Sec. 3.6. The foreground stars are mainly composed of three components: the halo stars, the thick disc component, and the thin disc component, for the line-of-sights of both Draco and Ursa Minor. To determine the prior, we use $v$ and $\log g$ information of the mock observation data. For the explanation, we categorize the dataset after the colour and ROI cuts into two types. 16

(i) the dataset with velocity cut.

(ii) the dataset with velocity \& $\log g$ cut.

We note that the velocity cut here implies masking the signal region ( $v_{\text {lower }}<v<v_{\text {upper }}$ ) to obtain the pure foreground samples. The goal is to determine the foreground shape in the dataset (ii). In the dataset (ii), although the dominant contribution is the halo component, the other components non-negligibly distort the shape of the foreground distribution. Therefore, we fit all components assuming that their velocity distribution can all be described by the Gaussian distribution function. However, because the disc distributions are located at around $\log g \gtrsim 4$, the number of the disc components after the $\log g$ cut becomes so small that the fit cannot converge well. Therefore, we first determine the shape of the thick/thin disc component using the data without $\log g$ cut (dataset (i)).

In the first fit, we fit the velocity distribution of the dataset (i) by the sum of the three Gaussians allowing all the parameters (normalizations, mean velocities, dispersions) free. As one can see in the Fig. A1, the fit can be successfully performed because the peaks of the three components are obvious. Then, assuming that the distributions of the thick/thin disc component do not change after the $\log g$ cut (except for their normalization), we use these mean velocities and dispersions to the next fit (dataset(ii)). ${ }^{17}$ Here we note that we cannot utilize the information of the halo distribution to the second fit because the halo distribution non-negligibly depends on the $\log g .{ }^{18}$

In the second fit, we again assume that the three components are the normal distribution and fit them to the dataset (ii) (with velocity and $\log g$ cut). In this fit, we constrain the shapes of the disc components by imposing the Gaussian prior of the mean velocities and the dispersions,

16 The number of the stars (including the member and foreground stars) after the colour and ROI cuts is $\sim 2000$ for the one-pointing case. Therefore, all the stellar data utilized in this analysis can be covered by the PFS observation (with 2394 fibres).

17 We have checked the $\log g$ dependence on these components and found that it is small.

18 The shapes of the halo component keep Gaussian in the range of interest, while its mean and width change with respect the $\log g$ value.
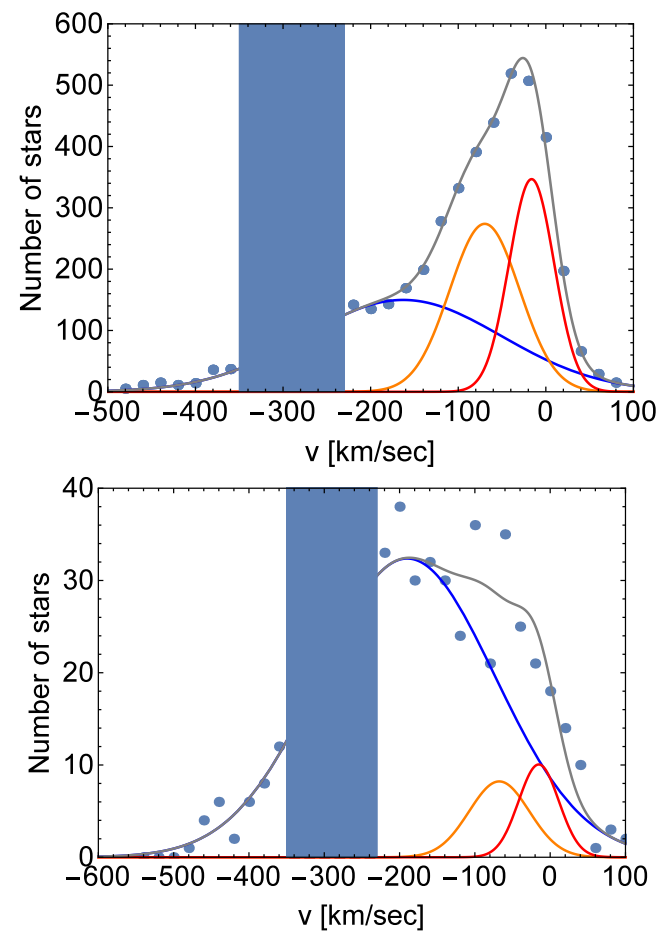

Figure A1. Foreground fit for the Draco 1 sample with $\theta_{R O I}=$ $1.3, i_{\max }=21$. The blue-shaded region is the signal region. The grey line shows the result of the fit and the blue, orange, red lines show the contribution from the halo, thick disc, and thin disc component respectively. The top panel is the result of the dataset (i) while the bottom is of the dataset (ii). See the text for more detail.

which are obtained from the first fit. The example of the fit is shown in the bottom panel of Fig. A1.

We finally note that the foreground curve in the signal region (the blue-shaded region in Fig. A1) is dominated by the halo component and therefore the prior function for the main fit in Sec. 3.6 can be described by the single Gaussian of the halo component.

\section{APPENDIX B: COMPARISON TO THE CONVENTIONAL METHOD}

In Sec. 4, we compare the results of our $J$-factor estimation with the 'Contaminated fit' analysis where we regard the foreground stars after the naive cuts in Sec.3.4 as the member stars. In the conventional analysis in Geringer-Sameth et al. (2015), the foreground stars are also treated as the member stars as in our 'Contaminated fit' to estimate the $J$-factor, although more sophisticated data cuts are applied so that the fraction of the member stars in the data set increases. In this section, we compare the results obtained by the conventional analysis (sophisticated extraction scheme + naive contaminated fit) with ours (naive cut + our likelihood fit).

Below, we first review the calculation process of the membership probability, giving the relation to our fit. We specifically consider the method adopted in Walker et al. (2009a) based on the Expectation Maximization (EM) al- 
gorithm. We then provide the results of the conventional method using the mock data obtained in Sec. 3.2.

\section{B1 Basics}

In contrast with our analysis, the conventional analysis assigns a membership probability $\tilde{p}\left(X_{i}\right)$ to each stars. Here $X_{i}$ denotes the information of $i$-th star (position, velocity, metalicity, etcetera). The $J$-factor is estimated by regarding all the stars with a given confidence level of $\tilde{p}\left(X_{i}\right)$, (e.g. $\left.\tilde{p}\left(X_{i}\right)>0.95\right)$, as member stars. Therefore, in the conventional way the robust estimation of $\tilde{p}\left(X_{i}\right)$ plays important role for the $J$-factor estimation.

If we could distinguish the members from the foregrounds, the membership probability would be given by the probability to find a member star at the specified distance, such as

$$
\tilde{p}\left(X_{i}\right) \equiv p\left(M_{i}=1 \mid X_{i}, \theta_{\mathrm{Mem}}, \theta_{\mathrm{FG}}, s\right),
$$

where the new observable $M_{i}$ takes a boolean value which indicates whether $i$-th star is a member $(M=1)$ or a foreground $(M=0)$, introduced for the convenience. ${ }^{19}$ The parameters $\theta_{\mathrm{Mem}}, \theta_{\mathrm{FG}}$ and $s$ are the free parameters describing the member and foreground distributions.

The explicit form of $\tilde{p}\left(X_{i}\right)$ can be derived from the fundamental likelihood $\mathcal{L}_{0} \equiv \prod_{i} p\left(M_{i}, X_{i} \mid \theta_{\mathrm{Mem}}, \theta_{\mathrm{FG}}, s\right)$ where we define

$$
\begin{aligned}
\ln \mathcal{L}_{0}=\sum_{i} M_{i} \ln \left(s f_{\mathrm{Mem}}\left(X_{i}\right)\right) & \\
& +\left(1-M_{i}\right) \ln \left((1-s) f_{\mathrm{FG}}\left(X_{i}\right)\right) .
\end{aligned}
$$

The explicit form of $\tilde{p}\left(X_{i}\right)$ is given by

$$
\tilde{p}\left(X_{i}\right)=\frac{s f_{\mathrm{Mem}}\left(X_{i}\right)}{s f_{\mathrm{Mem}}\left(X_{i}\right)+(1-s) f_{\mathrm{FG}}\left(X_{i}\right)} .
$$

We note that our likelihood $\mathcal{L}$ is equal to $p\left(X_{i}=\right.$ $\left.\left\{v_{i}, R_{i}\right\} \mid \theta_{\mathrm{Mem}}, \theta_{\mathrm{FG}}, s\right)=\prod_{i} \sum_{M_{i}=1,0} \mathcal{L}_{0}$.

In the conventional analysis, likelihood method is used to maximize $\prod_{i} p\left(X_{i / R_{i}} \mid R_{i}, \theta_{\mathrm{Mem}}, \theta_{\mathrm{FG}}, s\right)$ and the parameter at the maximum is used to evaluate $\tilde{p}\left(X_{i}\right)$. Here $X_{i / R_{i}}$ represents the stellar information except for its position. For this maximum search, Expectation Maximization (EM) algorithm is adopted, which enables us to estimate $\tilde{p}\left(X_{i}\right)$ incidentally through the recursive calculation based on $\tilde{\mathcal{L}} \equiv$ $\prod_{i} p\left(M_{i}, X_{i / R_{i}} \mid R_{i}, \theta_{\mathrm{Mem}}, \theta_{\mathrm{FG}}, s\right)$ instead of the direct maximization of its integration $\prod_{i} p\left(X_{i / R_{i}} \mid R_{i}, \theta_{\mathrm{Mem}}, \theta_{\mathrm{FG}}, s\right)=$ $\prod_{i} \sum_{M_{i}} \tilde{\mathcal{L}}$. Here, by using the expression in Eq.(B2), the likelihood $\tilde{\mathcal{L}}$ can be written as

$$
\begin{aligned}
\ln \tilde{\mathcal{L}} \equiv \sum_{i} M_{i} & \ln \left(\frac{f_{\mathrm{Mem}}\left(X_{i}\right)}{f_{\mathrm{Mem}}\left(R_{i}\right)} t\left(R_{i}\right)\right) \\
& +\left(1-M_{i}\right) \ln \left(\frac{f_{\mathrm{FG}}\left(X_{i}\right)}{f_{\mathrm{FG}}\left(R_{i}\right)}\left(1-t\left(R_{i}\right)\right)\right) .
\end{aligned}
$$

Here we introduce $f_{\mathrm{Mem}}\left(R_{i}\right) \equiv \int d X_{i / R_{i}} f_{\mathrm{Mem}}\left(X_{i}\right)$ and $f_{\mathrm{FG}}\left(R_{i}\right) \equiv \int d X_{i / R_{i}} f_{\mathrm{FG}}\left(X_{i}\right)$, for the convenience. $t\left(R_{i}\right) \equiv$ $s f_{\mathrm{Mem}}\left(R_{i}\right) /\left(s f_{\mathrm{Mem}}\left(R_{i}\right)+(1-s) f_{\mathrm{FG}}\left(R_{i}\right)\right)$ represents the member fraction of the stellar spatial distribution. Note that

19 We use notation $p(X \mid Y)$ for the posterior probability (or probability density function, p.d.f.) of $X$ after the observation of $Y$.
$f_{\mathrm{Mem}}(X) / f_{\mathrm{Mem}}(R)$ and $f_{\mathrm{FG}}(X) / f_{\mathrm{FG}}(R)$ corresponds to the velocity distribution function $\mathcal{G}\left[v ; v_{\mathrm{Mem}}, \sigma_{\text {l.o.s }}(R)\right]$ and $\mathcal{G}\left[v ; v_{\mathrm{FG}}, \sigma_{\mathrm{FG}}\right]$ of our analysis respectively.

In addition, the models of the distribution functions used in the conventional method are different from ours. First, no specific functional form of the spatial distribution functions $f_{\mathrm{Mem}}(R), f_{\mathrm{FG}}(R)$ are assumed, while we adopt the Plummer profile and linear increasing function of $R$ respectively. Rather, they only assume that $t\left(R_{i}\right)$ is monotonically decreasing function of $R$ and optimize it considering the value of $t\left(R_{i}\right)$ at each $R_{i}$ as a free parameter. Second, they use the velocity distribution functions defined by

$$
\begin{aligned}
\frac{f_{\mathrm{Mem}}(v, R)}{f_{\mathrm{Mem}}(R)} & =\mathcal{G}\left[v ; \tilde{v}_{\mathrm{Mem}}, \tilde{\sigma}_{\mathrm{Mem}}\right], \\
\frac{f_{\mathrm{FG}}(v, R)}{f_{\mathrm{FG}}(R)} & =\frac{1}{N_{\mathrm{Bes}}} \sum_{i=1}^{N_{\mathrm{Bes}}} \mathcal{G}\left[v ; \tilde{v}_{\mathrm{FG}}, \tilde{\sigma}_{\mathrm{FG}}\right],
\end{aligned}
$$

where $\tilde{v}_{\mathrm{Mem}}$ and $\tilde{\sigma}_{\mathrm{Mem}}$ are free parameters of the likelihood function $\tilde{\mathcal{L}}$, whereas $\tilde{v}_{\mathrm{FG}_{i}}, \tilde{\sigma}_{\mathrm{FG}}$ and $N_{\mathrm{Bes}}$ are fixed parameters achieved by the numerical simulation based on the Besançon model. We here stress that this assumption means that $\tilde{\sigma}_{\mathrm{Mem}}$ does not depend on $R$ and therefore the membership probability obtained by this process is biased to have a constant velocity dispersion curve. Moreover, in the conventional analysis, $f_{\mathrm{FG}}(v)$ is completely fixed by the numerical model while our distribution function has free parameters $v_{\mathrm{FG}}, \sigma_{\mathrm{FG}}$ in the Gaussian. We also note that the conventional analysis uses stars with a membership probability of a given confident level for the $J$-factor estimation. In this fit the systematic bias caused by the foreground contamination is not taken into account. This effect could be included in the fit by modifying the likelihood function given by Eq. (8) introducing weights from the membership probability for each star (Bonnivard et al. 2016). However, since the EM method only provides the parameter at the maximum of the likelihood, one cannot obtain the error of each membership probability. Therefore, the systematic uncertainty from this error is not included even in the modified fit. In our analysis, in contrast, we do not need to handle this systematic uncertainty because it is automatically included in the error bars obtained by the MCMC sampling method using our likelihood function.

\section{B2 $J$-factor estimation using mock data}

In this section, we give the results of the $J$-factor estimation by the conventional fit. We generate the mock data by the same process in Sec. 3.2. Here we do not impose any cuts to the data except for the color magnitude cut. Instead, the EM method given by Walker et al. (2009a) is applied to each mock using the information of $[\mathrm{Fe} / \mathrm{H}], \log g, v$ and $R$. The membership probability is assigned to each star after through the EM algorithm and the stars with the membership probability above $95 \%$ are extracted. Table B1 shows the number of the member and foreground stars after this process (averaged by the 50 mocks). The fraction of the foreground stars is less than $5 \%$ and better than those obtained by the naive cut process in Sec. 3.4 (see Table 4). This is because the information of the spacial distribution is used in the EM method, which is not involved in the cut process. Moreover, in the EM method, more accurate discrimination 


\begin{tabular}{|c|c|c|c|c|}
\hline dSph & $\theta_{\mathrm{ROI}}[$ degree $]$ & $i_{\max }[\mathrm{mag}]$ & $N_{\mathrm{Mem}}$ & $N_{\mathrm{FG}}$ \\
\hline \multirow[t]{4}{*}{ Draco $1 \& 2$} & 0.65 & 19.5 & 270 & 8 \\
\hline & & 21 & 900 & 11 \\
\hline & & 21.5 & 1140 & 12 \\
\hline & 1.3 & 21 & 920 & 22 \\
\hline \multirow[t]{4}{*}{ Ursa Minor } & 0.65 & 19.5 & 290 & 3 \\
\hline & & 21 & 1120 & 11 \\
\hline & & 21.5 & 1450 & 14 \\
\hline & 1.3 & 21 & 1140 & 16 \\
\hline
\end{tabular}

Table B1. The number of the member (foreground) stars with membership probability above $95 \%$ are given by $N_{M e m}\left(N_{F G}\right)$. 50 mocks are generated by the same procedure as Sec. 3.2 and the membership probability for each star is calculated by the EM method (see the text and Walker et al. (2009a) for the details).

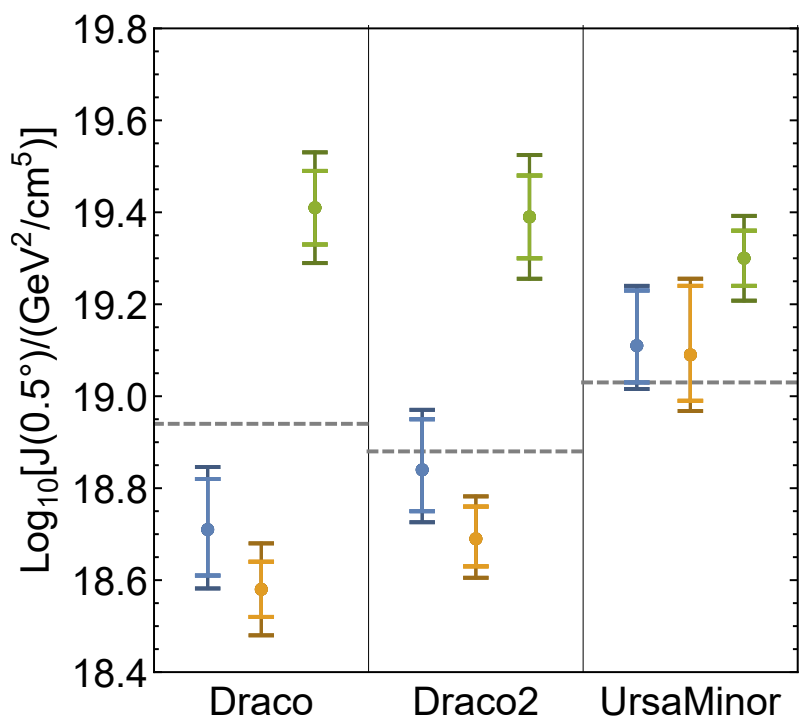

Figure B1. The $J$-factors obtained by the conventional method for the case of $i_{\max }=21$ with $\theta_{R O I}=0.65$ are plotted (orange bars). For comparison, $J$-factor estimation of 'Our fit' (blue bars) and 'Contaminated' (green bars) are also shown. The lighter error bars show the average of the $68 \%$ quantile, while the darker ones show the square root of the $68 \%$ quantiles and the standard deviation of the median values.

can be achieved by the weights from the $[\mathrm{Fe} / \mathrm{H}]$ and $\log g$ through the optimization of the means and widths of these Gaussian distributions.

Using this extracted data, we estimate the $J$-factor by the same method as 'Contaminated' case (in which, i.e., the data is fitted by using $f_{\mathrm{Mem}}$ in Eq.(8) accepting all the extracted star as the member star). We provide the result of the case of $i_{\max }=21$ with $\theta_{\mathrm{ROI}}=0.65$ in Fig. B1. The figure shows that even though the contamination is less than $5 \%$, mild systematic biases appear in the conventional method for Draco 1 and Draco 2 cases. This systematic bias decreases the median value of the $J$-factor, in contrast to the overestimation of the 'Contaminated' fit. This is because the distribution of the stars is different from the 'Contaminated' data. In the conventional approach, the constant velocity disper-
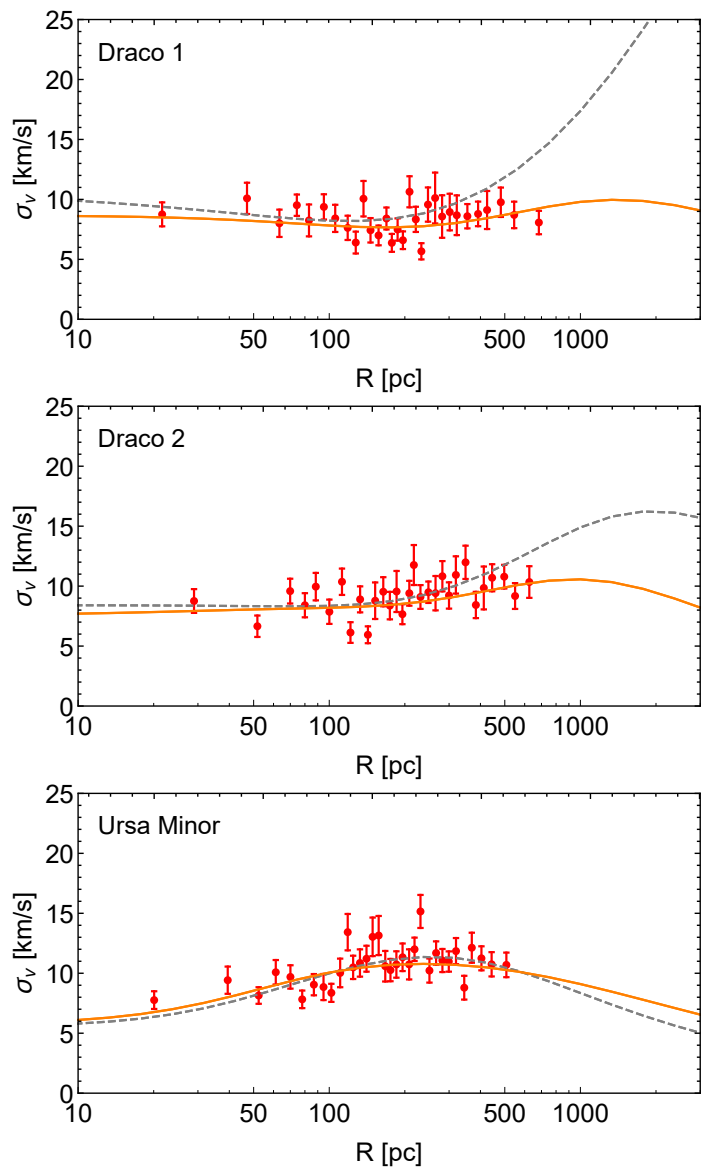

Figure B2. The dispersion curve of $\theta_{R O I}=0.65, i_{\max }=21$ case of Draco 1 (top), Draco 2 (middle), and Ursa Minor (bottom). The binned dispersions of the mock data after the EM selection are shown by the red dots with error bars. The orange line shows the dispersion curve of the best fit. The dashed line is obtained from the input parameter of the dSph dark matter halo.

sion is assumed and hence the extracted data can be biased to have the constant dispersion. This tendency is obvious for the two Draco cases, where the input dispersion curves largely increase at the outer region as shown in Fig. B2. We stress that this bias does not appear in our process because we adopt the range of the naive cuts wide enough in order not to distort the velocity distribution. ${ }^{20}$

Before closing this appendix, we comment on the ultrafaint dSphs case. Since the number of the member stars is much smaller than the classicals, the foreground contamination can be large even after the EM selection. Moreover, as discussed above, the conventional method has the bias of the constant velocity dispersion. In the ultra-faint dSphs, the parameter of the constant dispersion is controlled by the large foreground contamination and small member stars and therefore can often be misidentified. These effects can be a dominant hidden systematic uncertainties for the ultra-faint dSphs case, deriving a huge deviation

20 In the cut process, some fraction of the member stars is eliminated from the raw data by the naive $\log g$ cut. However, since $\log g$ cut is independent from the $r, v$ distribution of the member stars, this naive cut does not affect the dispersion curve. 


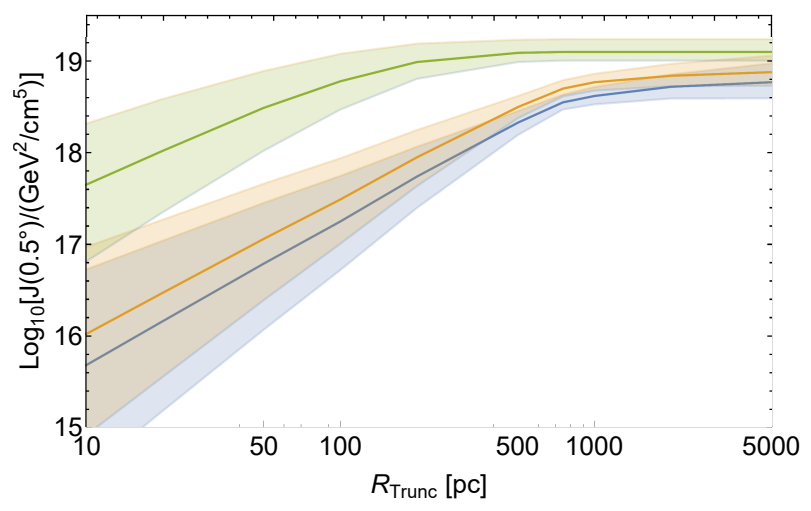

Figure $\mathbf{C} 1$. The dependence of the truncation radius for the $J$ factors for the case of $i_{\max }=21$ with $\theta_{R O I}=0.65$. The blue, orange and green lines show the $J$-factor median values of 50 mocks of Draco 1, Draco 2, and Ursa Minor respectively. The lighter bands show the averages of the $68 \%$ quantiles.

from the input $J$-factor. Due to the simultaneous fit of the member and foreground distribution including the $R$ dependence of the dispersion curve, our analysis will provide safer estimation especially for the ultra-faint dSphs, as demonstrated for classical dSphs in this paper. We will give detailed results for the ultra-faint dSphs case in the forthcoming work (Ichikawa et al. 2017).

\section{APPENDIX C: EFFECT OF THE \\ TRUNCATION RADIUS}

In our analysis, we set the truncation radius of the dark matter halo to 2000 pc. By using the MCMC samples obtained by our fit, we check the dependence of the truncation radius for the $J$-factor and the size of the error bars. Fig. C1 shows this dependence and we find that the fluctuation of the $J$-factor error is not largely affected by the truncation radius when it is above $1000 \mathrm{pc}$ and the error is minimized at around $800 \mathrm{pc}$.

This paper has been typeset from a $\mathrm{T}_{\mathrm{E}} \mathrm{X} / \mathrm{LAT} \mathrm{T}$ f file prepared by the author. 